\title{
EFFECTIVENESS OF MOTIVATIONAL INTERVIEWING AND GAMES ON ORAL HYGIENE OF CHILDRENAND ORAL HEALTH KNOWLEDGE, ATTITUDE AND BEHAVIOR OF MOTHERS: A Randomized Controlled Clinical Trial
}

\author{
Amany M.Nomair ${ }^{1}{ }_{B D S}$, Maha A. Hamza2 PhD,Wafaa E. Abdelazizz $P h D$.
}

\begin{abstract}
OBJECTIVES: To evaluate the effectiveness of motivational interviewing (MI) as well as games in changing oral health behaviors among preschool children in Edko administration, Beheira governorate, Egypt.

MATERIALS AND METHODS: The participating children, in this randomized controlled clinical trial, in 2018, were recruited from the 12 governmental preschools available within Edko administration of Beheira governorate, Egypt. A cluster random sampling strategy was used so that the contributors, mother/child dyads (pairs), in each nursery, were subsequently assigned to experimental group I (MI), experimental group II (MI+ gaming) or the control group (conventional dental health education (CE). The two outcomes, oral hygiene index simplified (OHI-S) for children and knowledge, attitude and practice of children's mothers were measured at base line and after six months follow-up. Paired t-test was used.

RESULTS: After six months follow-up, compared with the control group $(1.41 \pm 0.77)$, preschool children in experimental group I and II had significantly lower mean \pm SD of OHI-S $(0.69 \pm 0.61$ and $0.70 \pm 0.72), \mathrm{P}<0.001$ and significantly higher mothers' knowledge (26.98 \pm 2.90 and $25.75 \pm 2.52$ ), attitude (19.52 \pm 2.14 and $20.17 \pm 4.16)$ and practice scores $(9.75 \pm 2.05$ and $9.08 \pm 2.20)$, where $\mathrm{P}<0.05$.

CONCLUSIONS: Using MI as an oral health education tool was significantly more effective in promoting preschool children's oral hygiene and improving mothers' knowledge, attitude and oral health related practices than CE alone.

The protocol of the study was registered and posted on the ClinicalTrials.gov public website. ClinicalTrials.gov Identifier: NCT04258748.

KEYWORDS: Oral health education, motivational interviewing, game based education, oral health behaviors, preschool children.

RUNNING TITLE: effectiveness of motivational interviewing and game based education.
\end{abstract}

1 Bachelor of Dental Surgery, Faculty of Dentistry, Alexandria University, Egypt.

2. Professor of Dental Public Health, Department of Pediatric Dentistry and Dental Public Health, Faculty of Dentistry, Alexandria University, Egypt.

* Corresponding Author:

Email: Amani_nomair@yahoo.com

\section{INTRODUCTION}

The poor oral health of young children, below six years of age, negatively impacts their oral health outcomes, whether in childhood or throughout adult life (1).Conventional education (CE), whether conducted by professionals or disseminated via pamphlets, posters and media campaigns (2) maybe not enough to reach positive attitudes and favorable behaviors (3).Hence, behavior-change techniques, including face to-face counseling, have been tried and proven effective. They are considered an inherent component of an intervention that aims to alter and regulate behavior. An example is Motivational Interviewing (MI) (4), which is a patient-centered approach that focuses on building intrinsic motivation for behavior change by exploring and resolving conflicting emotions and uncertainty about health issues (5).The effectiveness of the MI approach in comparison to CE was compared in the study performed by Naidu et al. (4), who proved the superiority of the MI in improving parent/ caregiver oral health knowledge, attitudes and behaviors. However, Freudenthal and Bowen (6), reported no significant change regarding dental health as a result of MI intervention in comparison to CE for mothers. Digital games have been also acknowledged as beneficial medium to promote health and to provide an opportunity for interdisciplinary education (7). Aljafari et al (8), reported that oral health education using a computer game might be satisfactory and effective in improving oral health habits of children as CE.

No studies compared the effectiveness of MI in combination with games, directed to children, as an oral health educational intervention. Therefore, the present study aimed at exploring the effectiveness of both interventions; MI tailored to mothers and games directed to children, in changing oral health behaviors of mothers and improving oral hygiene of preschool children, in Beheira governorate. The null hypothesis of the current trial is assuming no significant differences between both interventions when compared with $\mathrm{CE}$ in changing the 
mothers' oral health knowledge, attitude and behavior and subsequent improvement oral hygiene of children, which is the primary outcome.

\section{MATERIALS AND METHODS}

The present study was a three parallel arms, randomized, controlled, clinical trial that was conducted among preschool children and their mothers, for a period of six months. The participating children were recruited from the twelve governmental nurseries available within Edko administration, Beheira governorate, Egypt.

Approval of the Research Ethics Committee of the Faculty of Dentistry, Alexandria University, (IRB NO: 00010556 IORG 0008839), was first sought before conduction of the study. Informed written consent was then obtained from each participating mother.

The minimal sample size was calculated based on a study that aimed to compare the effectiveness of conventional and "game-based" teaching on the oral health status of school children. Kumar et al.(9), reported a change in the mean \pm SD of debris index score from $1.30 \pm 0.35$ to $0.73 \pm 0.25$, in Flash cards and game group. Therefore, assuming an alpha error $=5 \%$ and a study power $=80 \%$, the minimal sample size was calculated to be 174 for the three groups.

The placement of nurseries in a particular study group was based on using computer generated list of random numbers (nurseries were numbered 1-12 and these numbers were randomly assigned to control group, experimental group I (MI) and experimental group II (MI + gaming), using cluster random sampling technique. Each group consisted of four preschool clusters. After fulfilling eligibility criteria, which included healthy children, of both sexes, whose age ranging from 4-6 years, with unfavorable oral health behavior and the child's mother agrees to give consent and join the study. Yet, a child was excluded in case of having a history of serious medical condition or special care needs, using any type of removable appliances and illiterate mothers were also excluded. The number of eligible mother/child dyads were 285. The children were then randomly assigned to one of the three study arms, in the allocation ratio $1: 1: 1$, using random allocation software in blocks of five (10) to select the study sample of 174 participants. The preschools' randomization sequence were written on identical sheets of paper and folded inside opaque envelopes that was sealed to be open only at the time of intervention implementation.

Invitations were prepared to be sent to 58 mothers per study group. In the control group, mothers were invited to attend a single educational lecture, which was delivered through a 15minutes, in a hall inside the nursery, on oral health. Mothers were given brief information about causes of dental caries and role of plaque, toothbrush size, frequency of sweet snacks, brushing technique, toothpaste and fluoride use as well as importance of regular dental visits. Participants were then shown a short educational video about proper tooth brushing method, available on the internet, coupled with oral hygiene instructions.

Regarding experimental group I, in each of the four MI counseling sessions that were delivered inside the nursery manager's office, the researcher approached the mothers in groups of five, via three phases, in 15-30 minutes, to establish rapport/ alliance, to identify the child's desired oral health and finally to reinforce the motivation for changing behavior. Reinforcement meetings were programmed as follows: second visit/session after one month, third visit after two months and the last visit after another two months. All the MI visits were finished in five months. On each occasion, the motivations sustaining the changes were reinforced, with the identification of problems and the adoption of solutions (4).

In experimental group II, a combination of MI for mothers and gaming for preschool children was delivered. The children were engaged in 'Happy Teeth, Healthy Kids' which is an android tablet game (11), in which the child helped the toy to follow the steps of brushing teeth. It was included in the MI session scheduled for the mothers. After finishing MI sessions, children were invited to play the android game. All participating children were assessed to measure OHI-S, which is the primary outcome, at baseline and followed after six months. Dental examination was performed on a comfortable chair and under day light. The WHO, light weight, ball ended periodontal probes and dental mirrors were used. Change in Oral health knowledge, attitudes and behaviors of the mothers was assessed, at baseline and followed after six months, using a structured questionnaire based on literature review(12).The demographic questions included information on the child's age and gender, education level of the parents and employment status of the guardian. Questions about mothers' oral health knowledge were mainly about causes of caries, plaque accumulation, snacking habits of the child, whether tooth brushing would prevent gingival inflammation, size of tooth brush and amount of toothpaste used for the child. Meanwhile, attitude questions enquired about relation of oral health to general health and quality of life, importance of oral care in prevention of dental problems, prevention of caries in primary teeth and first permanent molars, regular tooth brushing with fluoride toothpaste and regular dental checkup. Mothers were also asked about their oral health behavior such as frequency of brushing their children's teeth, whether they use fluoride toothpaste, number of snacking times and reasons for their children's last dental visit. Validating the questionnaire was established by six experts who are acquainted with the topic. The initial translation of the questionnaire from the English language to the Arabic language was made by a translator. The initial translation was back-translated to ensure the accuracy of the translation. The questionnaires were fulfilled, during interviewing between researcher and mothers. Inside the nurseries, at baseline and followed up after six months.

Statistical analysis was carried out using statistical package for social sciences (IBM SPSS Statistics for Windows, Version 22.0. Armonk, NY). The Paired t-test was employed to compare the mean scores knowledge, attitude and practice, before and after the intervention in each group. ANOVA test was used for comparing the three study groups and Post Hoc Test for comparing between the two experimental groups after the interventions. Wilcoxon signed ranks test was used for comparing oral hygiene of children before and after the intervention in each group, (significance level: $\mathrm{P} \leq 0.05$ ). Flow chart of research design 


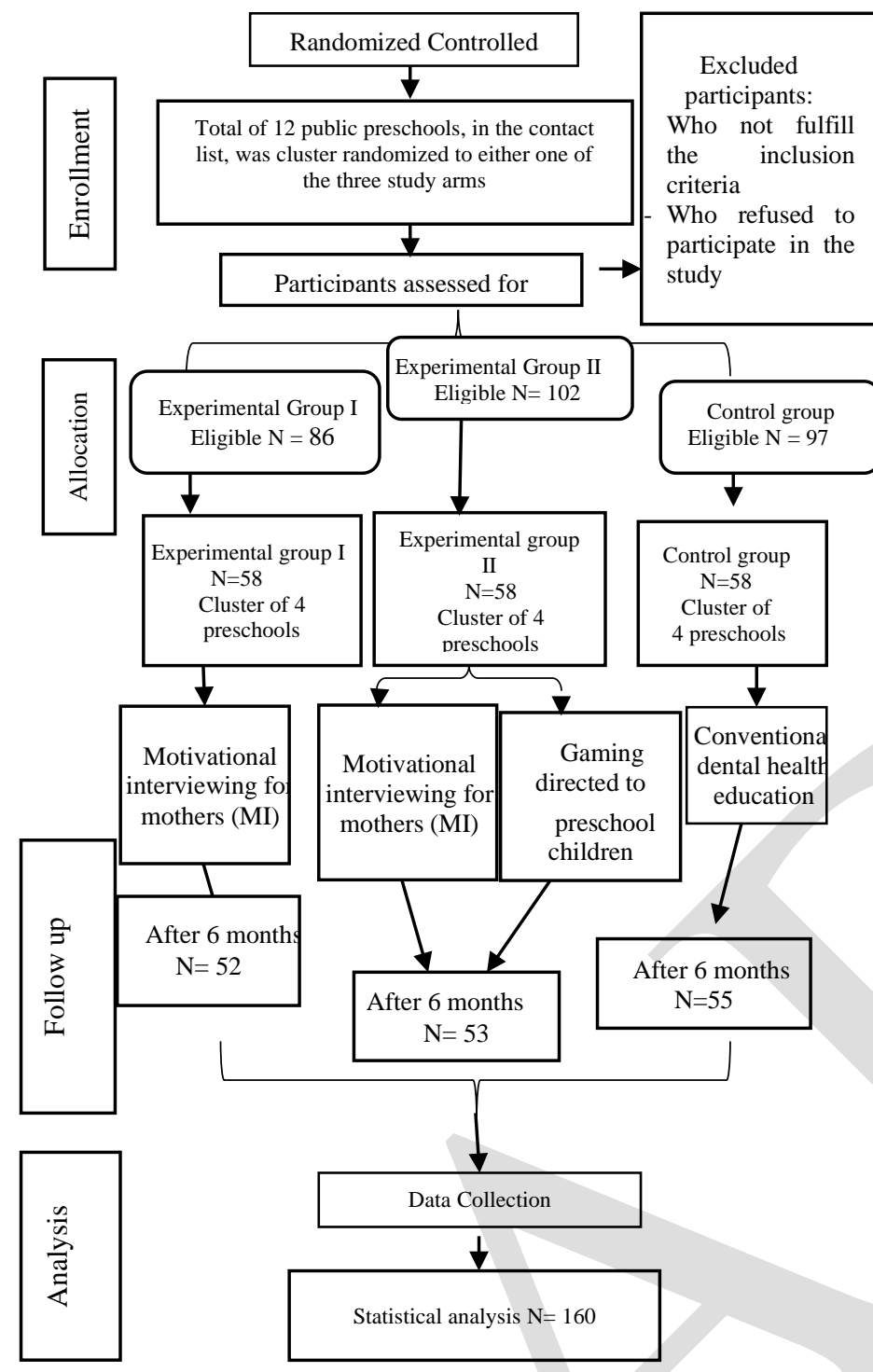

Figure 1: flow chart

\section{RESULTS}

Data in table (1) shows no significant differences regarding all the demographic variables of all three studied groups namely gender $(\mathrm{P}=0.542)$, age $(\mathrm{P}=0.165)$, mother's education $(\mathrm{P}=0.116)$, father's education $(\mathrm{P}=0.154)$ and employment status $(\mathrm{P}=0.763)$.

Table (2) shows the comparison between before and after intervention according to knowledge, attitude and practice scores in each group. In the control group; there was no significant difference according to the mothers' knowledge, attitude and practice scores before and after conventional education $(\mathrm{P}=0.062, \mathrm{P}=0.099$ and $\mathrm{P}=0.061)$. In experimental group I, a significant change in the mean \pm SD of knowledge score was detected before (21.29 \pm 2.91$)$ and after (26.98 \pm 2.90$)$ MI $(\mathrm{P}<0.001)$. A significantly better attitude score was also detected after MI $(\mathrm{P}<0.001)$, where the mean \pm SD of attitude score increased from $18.05 \pm 2.70$ to $19.52 \pm 2.14$, with a similar

Alexandria Dental Journal. Volume 46 Issue 2 Section C significant difference regarding practice scores before (7.04 \pm $1.98)$ and after $(9.75 \pm 2.05) \mathrm{MI}(\mathrm{P}<0.001)$. In experimental group II, there was a significant change in the mean \pm SD of knowledge score before (20.94 \pm 2.63$)$ and after $(25.75 \pm 2.52)$ $\mathrm{MI}$ and gaming $(\mathrm{P}<0.001)$, in the mean $\pm \mathrm{SD}$ of attitude scores before (19.52 \pm 2.14$)$ and after $(20.17 \pm 4.16) \mathrm{MI}$ and gaming $(\mathrm{P}<0.001)$ as well as in the mean practice scores before $(6.85$ $\pm 1.97)$ and after $(9.08 \pm 2.20) \mathrm{MI}$ and gaming $(\mathrm{P}<0.001)$. There was a significant difference among the three study groups regarding knowledge, attitude and practice scores after the interventions $(\mathrm{P}<0.001, \mathrm{P}<0.001$ and $\mathrm{P}=0.035)$. However, no significant difference was detected between both experimental groups regarding knowledge, attitude or practice scores after the interventions ( $\mathrm{P}=0.066, \mathrm{P}=0.575$ and $\mathrm{P}=0.297$ ).

Table (3) shows the comparison between before and after intervention according to OHI-S in each group of preschool children. In the control group; there was no significant difference before and after conventional education ( $\mathrm{P}=0.107)$, where the mean \pm SD of OHI-S changed from $1.66 \pm 0.82$ to $1.41 \pm 0.77$. Regarding experimental group I, a significantly lower mean OHI-S was detected after intervention (0.690 $\pm 0.61, \mathrm{P}<0.001)$, similarly, the experimental group II showed significantly lower mean \pm SD of OHI-S after intervention $(0.70 \pm 0.72, \mathrm{P}<0.001)$. There was a significant difference between the three study groups regarding OHI-S $(\mathrm{P}<0.001)$.However, there was no significant difference between experimental group I and II according to OHI-S (P $=0.836$ ).

Table (1): Distribution of the three studied groups according to demographic data 


\begin{tabular}{|c|c|c|c|c|c|c|c|c|}
\hline \multirow{3}{*}{ Demographic data } & \multirow{2}{*}{\multicolumn{2}{|c|}{$\begin{array}{l}\text { Control } \\
(\mathrm{n}=55)\end{array}$}} & \multicolumn{4}{|c|}{ 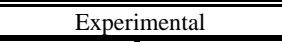 } & \multirow{3}{*}{$\begin{array}{l}\text { Test of } \\
\text { Sig. }\end{array}$} & \multirow{3}{*}{ P } \\
\hline & & & \multicolumn{2}{|c|}{$\begin{array}{c}\text { Experiment } \\
\text { al group I } \\
(\mathrm{n}=52)\end{array}$} & \multicolumn{2}{|c|}{\begin{tabular}{|c|} 
Experimenta \\
l II group \\
$(\mathrm{n}=53)$ \\
\end{tabular}} & & \\
\hline & No. & $\%$ & No. & $\%$ & No. & $\%$ & & \\
\hline \begin{tabular}{|l} 
Gender of the child \\
Boys \\
Girls
\end{tabular} & $\begin{array}{l}27 \\
28 \\
\end{array}$ & $\begin{array}{l}49.1 \\
50.9 \\
\end{array}$ & $\begin{array}{l}27 \\
25 \\
\end{array}$ & \begin{tabular}{|l|}
51.9 \\
48.1 \\
\end{tabular} & $\begin{array}{l}22 \\
31 \\
\end{array}$ & \begin{tabular}{|l|}
41.5 \\
58.5
\end{tabular} & $\begin{array}{c}\chi^{2}= \\
1.226\end{array}$ & 0.542 \\
\hline $\begin{array}{l}\text { How old is your child } \\
\text { today? (years) } \\
\text { Mean } \pm \text { SD. }\end{array}$ & 4.78 & \pm 0.76 & 4.71 & \pm 0.72 & 4.53 & \pm 0.64 & $\begin{array}{c}\mathrm{F}= \\
1.820\end{array}$ & 0.165 \\
\hline $\begin{array}{l}\text { What level of } \\
\text { education have you } \\
\text { completed? } \\
\text { Read and write } \\
\text { Primary school } \\
\text { completed } \\
\text { Secondary school } \\
\text { completed } \\
\text { College/university } \\
\text { completed }\end{array}$ & $\begin{array}{l}10 \\
16\end{array}$ & $\begin{array}{r}18.2 \\
29.1 \\
45.5 \\
\\
7.3\end{array}$ & \begin{tabular}{|c}
6 \\
16 \\
29 \\
1
\end{tabular} & \begin{tabular}{|c|}
11.5 \\
30.8 \\
55.8 \\
1.9
\end{tabular} & $\begin{array}{l}16 \\
14 \\
18\end{array}$ & \begin{tabular}{|c}
30.2 \\
26.4 \\
34.0 \\
9.4
\end{tabular} & $\begin{array}{c}\chi^{2}= \\
10.034\end{array}$ & $\begin{array}{l}{ }^{\mathrm{MC}} \mathrm{p}= \\
0.116\end{array}$ \\
\hline $\begin{array}{l}\text { What level of } \\
\text { education the father } \\
\text { completed } \\
\text { Illiterate } \\
\text { Read and write } \\
\text { Primary school } \\
\text { completed } \\
\text { Secondary school } \\
\text { completed } \\
\text { College/university } \\
\text { completed }\end{array}$ & $\begin{array}{c}0 \\
9 \\
14\end{array}$ & $\begin{array}{r}0.0 \\
16.4 \\
25.5 \\
54.5 \\
3.6\end{array}$ & $\begin{array}{c}1 \\
13 \\
12 \\
25 \\
1\end{array}$ & $\begin{array}{r}1.9 \\
25.0 \\
23.1 \\
48.1 \\
\\
1.9\end{array}$ & $\begin{array}{c}0 \\
20 \\
13\end{array}$ & $\begin{array}{r}0.0 \\
37.7 \\
24.5 \\
32.1 \\
5.7\end{array}$ & $\begin{array}{c}\chi^{2}= \\
10.847\end{array}$ & $\begin{array}{l}\mathrm{MC}= \\
0.154\end{array}$ \\
\hline $\begin{array}{l}\text { What is the } \\
\text { employment Status } \\
\text { of father or guardian } \\
\text { Employed for wages/ } \\
\text { salary } \\
\text { Self-employed } \\
\text { Unable to work } \\
\end{array}$ & $\begin{array}{r}37 \\
4 \\
\end{array}$ & $\begin{array}{r}25.5 \\
67.3 \\
7.3 \\
\end{array}$ & $\begin{array}{c}18 \\
31 \\
3 \\
\end{array}$ & $\begin{array}{r}34.6 \\
59.6 \\
5.8 \\
\end{array}$ & $\begin{array}{c}30 \\
5 \\
\end{array}$ & $\begin{array}{r}34.0 \\
56.6 \\
9.4 \\
\end{array}$ & $\begin{array}{c}\chi^{2}= \\
1.992\end{array}$ & $\begin{array}{l}{ }^{M C} \mathrm{p}= \\
0.763\end{array}$ \\
\hline
\end{tabular}

$\chi^{2}$ : Chi square test MC: Monte Carlo $\quad F$ : $F$ for ANOVA test

$\mathrm{P}$ : P value for comparing between the studied groups

*: Statistically significant at $\mathrm{P} \leq 0.05$

Experimental group I: Motivational Interviewing

Experimental group II: MI and gaming

Table (2): Comparison between the three studied groups of mothers before and after intervention according to knowledge, attitude and practice scores

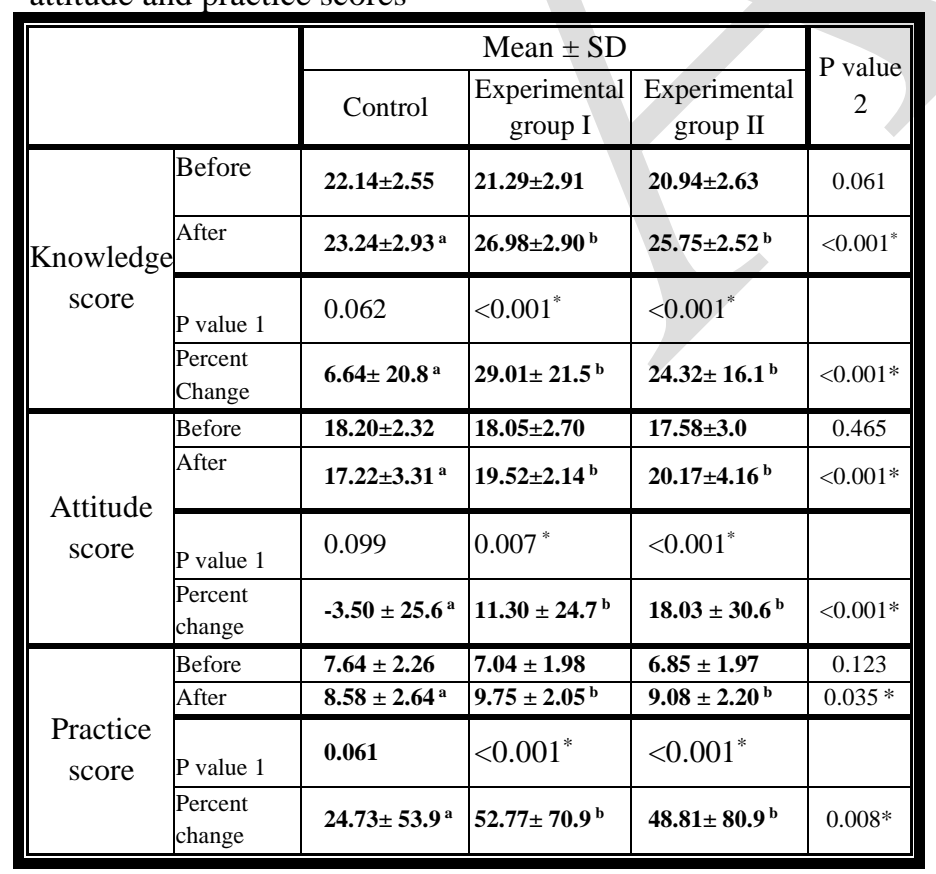

$\mathrm{P}$ value 1: $\mathrm{P}$ value for Paired t-test comparing before and after values in each group

$\mathrm{P}$ value 2: $\mathrm{P}$ value for ANOVA test comparing the 3 groups

a, b: different letters denote statistically significant differences using

Tukey post-hoc test

*: Statistically significant at $\mathrm{P} \leq 0.05$

Table (3): Comparison between the three studied groups of children before and after intervention according to oral hygiene index simplified

\begin{tabular}{|c|c|c|c|c|c|}
\hline & \multicolumn{3}{|c|}{ Mean \pm SD } & \multirow[b]{2}{*}{$\begin{array}{c}\text { P value } \\
2\end{array}$} \\
\hline & & Control & $\begin{array}{c}\text { Experimental } \\
\text { group I }\end{array}$ & $\begin{array}{c}\text { Experimental } \\
\text { group II }\end{array}$ & \\
\hline \multirow{4}{*}{$\begin{array}{l}\text { Oral } \\
\text { hygiene } \\
\text { index } \\
\text { (OHI-S) }\end{array}$} & Before & $1.66 \pm 0.82$ & $1.68 \pm 0.68$ & $1.69 \pm 0.59$ & 0.965 \\
\hline & After & $1.41 \pm 0.77^{a}$ & $0.69 \pm 0.61^{b}$ & $0.70 \pm 0.72^{b}$ & $<0.001 *$ \\
\hline & $P$ value 1 & 0.107 & $<0.001^{*}$ & $<0.001^{*}$ & \\
\hline & $\begin{array}{l}\text { Percent } \\
\text { Change }\end{array}$ & $11.0 \pm 136.8^{a}$ & $47.95 \pm 64.6^{b}$ & $57.59 \pm 40.2^{b}$ & $0.02 *$ \\
\hline
\end{tabular}

$\mathrm{P}$ value 1: $\mathrm{P}$ value for Wilcoxon signed ranks test comparing before and after values in each group

$\mathrm{P}$ value 2: $\mathrm{P}$ value for Kruskal Wallis test comparing the 3 groups a, b: different letters denote statistically significant differences using Dunn's post-hoc test

*: Statistically significant at $\mathrm{p} \leq 0.05$

\section{DISCUSSION}

Motivational interviewing may prompt changes in the knowledge, attitudes and oral hygiene practices of children's caregivers leading to healthy behaviors. This was, indeed, proved throughout the results of the current study, that showed significantly lower mean scores of OHI-S among children in both experimental groups, as well as significantly better mean knowledge, attitude and practice scores among mothers of experimental group I (MI) and II (MI and gaming), after intervention, compared to the control group, which safely leads to the rejection of the null hypothesis.

Actually, the MI sessions built a good relationship between the counsellor and mothers based on respect and confidence, which resulted in significant improvement in mothers' knowledge, attitude and behavior before and after the interventions. Meanwhile, CE did not achieve the same level of success as imparting knowledge, for the first time, needs new tools of communication and persistent follow-up process. In agreement with the current results, Naidu et al. (4), in Trinidad, proved the significant superiority of the MI in improving parent/ caregiver oral health knowledge, attitudes and behaviors. The design of the study of Naidu et al. (4), was a cluster randomized controlled trial. Seventy nine parents /caregivers were randomly assigned to an experimental or control group. The experimental group received a session on dental health using an MI approach, in addition to two telephone calls follow-ups, as part of the MI protocol. The control group received session using CE. The effectiveness of the MI approach was compared to the CE and proved to be superior in improving parents' oral health knowledge, 
attitudes and behaviors after four months follow-up. While similar studies proved the superiority of MI in comparison with CE (13-16), there are others which still contradict the current findings.

In disagreement with the current study, Freudenthal and Bowen (6), in Southeast Idaho, found no clinically significant change in valuing dental health as a result of MI intervention with the mothers. In this study, Freudenthal and Bowen (6), mothers in the control group received CE, while mothers, in the experimental group, received MI counseling sessions, yet, the follow-up was only by telephone calls and pretest and posttest questionnaires for mothers were completed, four weeks apart. In the current study, on the other hand, questionnaires for mothers were compared at base line and after six months follow-up and the MI follow-up sessions were achieved through face to face meetings. Moreover, the researcher had a greater opportunity to directly communicate with mothers, thus emphasizing the transfer of knowledge, eliminating contradictions and solving the problems facing them, which is not in agreement with the latter study. Brand et al.(17),similarly found significant decrease, over time, for bleeding on probing, plaque index and pocket depths for both MI and CE groups. However, differences in clinical parameters between groups were not evident at either six or twelve weeks follow up, which is not in agreement with the current findings where significant and sustained decrease in OHI-S scores were detected six months after intervention. Again this could be attributed to the benefits of MI sessions that focused on changing the internal thoughts and beliefs of the mothers regarding the importance of their children's oral health, in addition to the attempts to resolve any doubts or enquiries about health issues via the reinforcement sessions throughout the 6 months follow-up period. Although, the children in experimental group II were very interactive and motivated in the android game yet, reinforcing MI with game based oral health education did not yield significant improvement neither regarding oral health knowledge, attitude and practices of mothers nor oral hygiene of children of both experimental groups. Accordingly, the MI as an oral health educational technique directed to children's caregivers proved its effectiveness on its own irrelevant to the indulgence of their children in the educational process. In agreement with the current results, Aljafari et al (8), conducted a randomized controlled trial in order to assess the effect of video games and $\mathrm{CE}$ on the oral health knowledge, dietary and oral hygiene habits of 4- to 10-year-old children in UK. Children in both groups showed significant improvement in recognition of unhealthy foods immediately post-education $(\mathrm{P}<0.001)$. After three months, fifty-five per cent of all participants reported improvements in reducing sweetened drinks $(\mathrm{P}=$ 0.019). Also, children reported twice-daily tooth brushing, with no significant differences between the two groups.

Oral health education based on MI can offer an effective educational strategy especially in a population of underserved individuals and dental practitioners should be encouraged to attend continuing education courses about innovative health education strategies such as MI in order to enhance positive health behaviors of their patients. Yet, despite the positive results of the current trial, there were some problems facing the researcher, especially in this multi-component educational program, which required the teachers' help in organizing the sessions and providing the children with the android games. Hence, presence of trained assistants is crucial to be more focused on the phases of MI process including rapport, motivation for change and initiative for change. Furthermore, mothers' age should have been taken into consideration in the statistical analysis since it might be considered a confounding variable that would have affected the final outcomes of the study.

Also, there is a need for further studied among other socioeconomic societies in order to generalize the results and offer an effective strategy for oral health professionals to increase oral health knowledge, improve oral health attitude and facilitate oral health behavior change, for both the individuals with low socioeconomic status as well as for the general population.

Conclusion

Using $\mathrm{MI}$ as an oral health education tool was significantly more effective in promoting preschool children's oral hygiene and improving mothers' knowledge, attitude and oral health related practices than conventional education alone. Incorporation of games entails further studies to detect its effect.

\section{CONFLICT OF INTEREST}

The authors declared that they have no conflicts of interest.

\section{REFERENCES}

1. Kawashita Y, Kitamura M, Saito T. Early childhood caries. Int J Dent 2011; 2011:725320.

2. Kapoor V, Gupta A, Arya V. Behavioral changes after motivational interviewing versus traditional dental health education in parents of children with high caries risk: Results of a 1-year study. J Indian Soc Pedod Prev Dent 2019; 37:192-7.

3. D'Cruz A, Aradhya S. Impact of oral health education on oral hygiene knowledge, practices, plaque control and gingival health of 13-to 15-year-old school children in Bangalore city. Int J Dent Hyg 2013; 11: 126-33.

4. Naidu R, Nunn J, Irwin JD. The effect of motivational interviewing on oral healthcare knowledge, attitudes and behaviour of parents and caregivers of preschool children: an exploratory cluster randomised controlled study. BMC Oral Health 2015; 15:101.doi: 10.1186/s12903-015-0068-9.

5. Resnicow K, McMaster F. Motivational Interviewing: moving from why to how with autonomy support. Int J Behav Nutr Phys Act 2012; 9:19. doi:10.1186/1479-5868-9-19.

6. Freudenthal JJ, Bowen DM. Motivational interviewing to decrease parental risk-related behaviors for early childhood caries. J Dent Hyg 2010; 84:29-34.

7. Haaranen A, Rissanen $T$, Laatikainen $T$, Kauhanen J. Digital and video games in health promotion: Systematic review of games and health behavior. FinJeHeW 2014; 15:153-6.

8. Aljafari A, Gallagher JE, Hosey MT. Can oral health education be delivered to high-caries-risk children and their parents using a computer game? - A randomised controlled trial. Int J Paediatr Dent 2017; 27:476- 85.

9. Kumar Y, Asokan S, John B, Gopalan T. Effect of conventional and game-based teaching on oral health 
status of children: a randomized controlled trial. Int J Clin Pediatr Dent 2015; 8:123-6.

10. Saghaei M. Random allocation software for parallel group randomized trials. BMC Med Res Methodol 2004; 4:26.doi: 10.1186/1471-2288-4-26

11. TabTale. Happy Teeth, Healthy Kids. Available at: http://www.tabtale.com/app/happy-teeth/.

12. Gao J, Ruan J, Zhao L, Zhou H, Huang R, Tian J. Oral health status and oral health knowledge, attitudes and behavior among rural children in Shaanxi, western China: a cross-sectional survey. BMC Oral Health 2014; 14:144.doi:10.1186/1472-6831-14-144

13. González-Del-Castillo-McGrath M, Guizar-Mendoza JM, Madrigal-Orozco C, Anguiano-Flores L, Amador-Licona N. A parent motivational interviewing program for dental care in children of a rural population. J Clin Exp Dent 2014; 6: 524-9.
14. López-Jornet P, Fabio CA, Consuelo RA, Paz AM. Effectiveness of a motivational-behavioural skills protocol for oral hygiene among patients with hyposalivation. Gerodontology 2014; 31:288-95.

15. Mohammadi TM, Hajizamani A, Bozorgmehr E. Improving oral health status of preschool children using motivational interviewing method. Dent Res J 2015; 12:476-81.

16. Wu L, Gao X, Lo ECM, Ho SMY, McGrath C, Wong MCM. Motivational interviewing to promote oral health in adolescents: a randomized controlled trial. J Adolesc Health 2017; 61:378-84.

17. Brand VS, Bray KK, MacNeill S, Catley D, Williams K. Impact of single-session motivational interviewing on clinical outcomes following periodontal maintenance therapy. Int J Dent Hyg 2013; 11:134-41. 